\title{
Un estudio cualitativo sobre convivencia escolar: El Bullying desde la perspectiva de las Victimas
}

A qualitative study on school coexistence: Bullying from the perspective of Victims

Recibido el 29 de setiembre del 2017, aceptado el 10 de noviembre del 2017

\author{
Horna Calderón, Víctor \\ victor.hornac@usil.pe \\ Universidad San Ignacio de Loyola - Perú \\ ORCID: 0000-0002-3071-5491
}

Para referenciar este artículo: Horna, V. (2017). Un estudio cualitativo sobre convivencia escolar: El Bullying desde la perspectiva de las Víctimas. Conciencia EPG, 2(2), 46-56.

\section{Resumen}

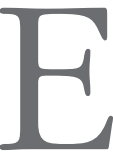
1 presente es un estudio de carácter exploratorio acerca de las cogniciones que el estudiante víctima de bullying tiene como explicación a esta forma de agresión que sufre. El principal objetivo fue determinar qué tipo de explicaciones ofrecen estos estudiantes frente al bullying, que hace que permanezcan en la situación de víctima, sin intentar modificar su condición. Se desarrolló bajo una metodología cualitativa de investigación, cuyos participantes fueron seleccionados de manera participativo-intencional, mediante la observación y entrevista como técnicas de recolección de datos y el programa ATLAS.TI para su análisis e interpretación. Como resultados se halló que los estudiantes víctimas de bullying, reconocen haberla sufrido en alguna oportunidad, pero muy pocos aceptan ser víctimas, buscando siempre alguna justificación, las cuales van desde la resignación momentánea hasta la esperanza de un final feliz como consuelo. Esta investigación representa una aproximación inicial al estudio del pensamiento de las victimas frente al bullying.

Palabras clave: bullying, convivencia, cognición, agresión, víctima.

\section{Summary}

he present is an exploratory study about the cognitions that the student victim of bullying has as an explanation to this form of aggression that suffers. The main objective was to determine what kind of explanations these students offer in the face of bullying, which makes them remain in the victim situation, without trying to modify their condition. It was developed under a qualitative research methodology, whose participants were selected in a participatory-intentional manner, through observation and interview as data collection techniques and the ATLAS.TI program for its analysis and interpretation. As results were found that students victims of bullying, acknowledge having suffered at some time, but very few accept to be victims, always looking for some justification, 
which range from momentary resignation to the hope of a happy ending as consolation. This research represents an initial approach to the study of victims' thinking in the face of bullying.

Key words: bullying, coexistence, cognition, aggression, victim.

\section{Introducción}

Todos estaremos de acuerdo que la convivencia escolar debe ser entendida como uno de los elementos de mayor importancia dentro del proceso de enseñanza aprendizaje cuyo objetivo es la adquisición del conocimiento por parte del individuo, especialmente cuando la asistencia al centro educativo y la preocupación o interés por aprender está estrechamente vinculado a la tranquilidad o motivación que el ambiente o clima escolar les brinde. El Clima social escolar (Giraldo \& Mera, 2000) contribuye a la socialización, a la autodeterminación y a la adquisición de responsabilidad por parte del estudiante, favoreciendo así la convivencia en el colegio y, por tanto, el desarrollo de la personalidad.

Es la escuela uno de los primeros espacios, dentro del cual el individuo forma y desarrolla su persona, en ella continuamente establece y fortalece relaciones interpersonales alimentadas desde el hogar y es este uno de los principales factores para el correcto desarrollo del mismo. Por ende, siendo la Escuela, el lugar dónde vamos a desarrollarnos académica, social, psicológica y culturalmente, debería por lo menos proporcionarnos un ambiente social apropiado para ello. Teniendo en cuenta que "la Institución Educativa, como comunidad de aprendizaje es la primera y la principal Instancia de Gestión del Sistema Educativo Descentralizado" (Ley General de Educación: Art. 66).

Lamentablemente existen factores vinculados con la violencia y sus diferentes manifestaciones, que al incubarse en la escuela afectan directamente la convivencia en la misma y por ende los objetivos que ella pretende. Uno de los problemas de mayor preocupación en nuestra sociedad contemporánea es el de la violencia, ejercida de manera individual o grupal, sea bajo razones justificables o no, como un reclamo, para cambiar actitudes, alcanzar objetivos e incluso como una forma de diversión. Dentro de estas manifestaciones, la violencia en la escuela es un problema tan antiguo como la misma educación, ésta se pone de manifiesto como un comportamiento que dificulta la convivencia entre los estudiantes generando no sólo efectos cada vez más nocivos sino también formas de aparición más sofisticadas y menos evidentes para autoridades, profesores y especialmente padres de familia.

Los fenómenos de violencia entre niños y jóvenes despiertan preocupación social y concentran cada vez más atenciones y esfuerzos educativos, debido principalmente a los efectos perjudiciales que ella provoca sobre el desempeño social a lo largo de la vida. Según Avilés (2001), las consecuencias que los comportamientos agresivos tienen sobre las personas que los padecen, e incluso, sobre quienes los llevan a cabo, son muy graves, en especial cuando se producen a edad temprana ya que dejan secuelas para el resto de la vida.

Una forma de violencia particular que afecta las relaciones interpersonales de los estudiantes en las escuelas, se viene estudiando últimamente como un fenómeno diferenciado bajo diversas modalidades, es el llamado bullying, palabra inglesa que se emplea para denominar los procesos de intimidación y victimización entre iguales, esto es, entre estudiantes compañeros de aula o de centro escolar (Ortega \& Mora-Merchán, 1997), los que se realizan cruelmente, con el objetivo de amedrentar, intimidar, tiranizar y someter emocionalmente a la víctima con vistas a obtener algún resultado favorable para los acosadores o satisfacer una necesidad de dominar y agredir a los otros estudiantes (Carozzo, 2010).

Diversos autores han intentado explicar el fenómeno del Bullying a partir de los factores sociales, educativos o de los participantes, especialmente el agresor o acosador, (Smith \& 
Thompson, 1991; Monjas,1992; Fernández, 1996; Ortega \& Mora-Merchán, 1996; Olweus,1998; Díaz, 2000; Avilés, 2001; Cerezo, 2001; Del Barrio, Montero, Gutiérrez, \& Fernández, 2003; Ortega, \& Del Rey, 2003; Piñuel \& Oñate, 2007; Oliveros, Figueroa, Mayorga, Cano, Quispe, \& Barrientos, 2009; Benites, Carozzo, Horna, Palomino, Salgado, Uribe \& Zapata, 2012; Carozzo, Benites, Zapata, \& Horna 2012, entre otros). En el presente estudio intentaremos abordarlo a partir de la concepción y perspectiva de las víctimas que lo sufren, sus opiniones, justificaciones, esperanzas y otras percepciones.

Teniendo presente estas consideraciones, nos propusimos realizar una investigación de tipo cualitativo, en un colegio particular mixto de educación secundaria, del distrito de san Borja en Lima - Perú. Siendo el Objetivo Conocer las cogniciones y explicaciones de los estudiantes víctimas de Bullying en una escuela, que permiten que permanezcan bajo dicha condición.

Creemos que, la problemática del bullying en la escuela, sólo podrá ser enfrentada en la medida que se conozca la forma en que se presenta y de qué manera afecta a los estudiantes, así se podrá realizar una sensibilización más realista con los participantes permitiendo que tomen un papel más activo en la prevención o intervención en el medio escolar específico, de acuerdo con las características de la población, comprometiendo de esta manera tanto a maestros, padres de familia, autoridades y obviamente a los propios estudiantes.

\section{Método}

La presente investigación representa un Estudio Cualitativo, de Tipo Descriptivo - Narrativo, sobre el comportamiento y las razones o explicaciones que hacen que un grupo de adolescentes víctimas de bullying permanezcan en dicha situación. Se utilizó un Diseño Narrativo a fin de identificar y clasificar las situaciones de bullying, así como para recoger la información relevante que permitiría su categorización, análisis e interpretación posterior acerca de las justificaciones, razones y otras cogniciones con las que los estudiantes victimizados explican la situación de maltrato que viven en el colegio.

Participantes: La población total fue 12 estudiantes pertenecientes a las aulas de $1^{\circ}$ a $5^{\circ}$ año de Secundaria del Centro Educativo Particular del distrito de san Borja, en Lima-Perú. Esta muestra fue seleccionada debido a que los niños manifestaron haber sufrido maltrato alguna vez, lo cual constituyó la muestra inicial de estudio, de ellos 3 eran de sexo femenino y 9 de sexo masculino de entre 12 a 16 años de edad. Fue seleccionada de manera intencionada, debido a las facilidades que ofrecía para la investigación, aunque solicitó mantenerse en el anonimato por razones de tipo empresarial. El rasgo en común resultó ser de hijos de padres que trabajan hasta muy tarde, casi no los ven durante el día y viven con abuelitos u otros parientes. Todos ellos pertenecen a una clase socioeconómica mediaalta, pueden satisfacer sus necesidades básicas y gozan de comodidades, pero sin ostentaciones.

Instrumentos: Se consideraron instrumentos de recogida de datos a:

Ficha Socio demográfica: Donde se consignan los datos de filiación y otras características particulares, que permite caracterizar a los sujetos del estudio y sus condiciones familiares.

Cuestionario de Convivencia y Maltrato (CCM): Es un cuestionario de cinco preguntas de elección forzada y opción múltiple en sus respuestas, que pretende discriminar el tipo de convivencia o maltrato a partir de la propia percepción del estudiante.

Formato de Observación: A fin de pautar las conductas, que deberán registrarse para recoger los aspectos importantes para la investigación. Consistía en una hoja previamente diseñada que señalaba las conductas y situaciones posibles a partir de lo cual se podía ir analizando posibles situaciones agresivas y de intimidación relacionadas con la investigación. 
Guía de Entrevista - Cuestionario: Considera algunas preguntas cerradas y/o que permitirán dirigir la entrevista hacia los objetivos de la investigación. Estaba basado en el Cuestionario INSEBULL 2007 de José María Avilés y Juan Antonio Elices (España) y adaptado a la realidad del Centro educativo y la muestra estudiada.

Programa de Análisis Cualitativo asistido por Computadora - ATLAS TI.: Un software diseñado para el análisis cualitativo de datos, que permite la organización sistemática y creativa de la información recopilada.

Procedimiento: Previamente se realizó una charla con los estudiantes del colegio, con la finalidad de motivar su participación, en ella se les daba a conocer los objetivos de la investigación y asimismo se garantizaba el anonimato de los participantes. Luego se procedió a encuestarlos en sus aulas, mediante un cuestionario de cinco preguntas, asegurando que su participación sería muy confidencial. Estas entrevistas permitieron un mayor acercamiento con aquellos estudiantes que estaban siendo victimizados, sin embargo, no todos reconocían su condición como tal, un requisito para cumplir con los objetivos de la investigación, lo que redujo la muestra en casi un $60 \%$; sólo entonces, previo consentimiento expreso, se procedió a realizar un análisis más profundo a nivel cualitativo, acerca de la manera cómo vienen siendo afectados, las razones o explicaciones que pudieran tener al respecto y qué soluciones pudieran darse al problema. En ese segundo procedimiento, se realizaron observaciones directas participativas y no participativas, en el escenario de la investigación, así como entrevistas y encuestas que permitieran captar la percepción de las víctimas sobre la problemática -bullying- y su explicación o elaboración cognitiva al respecto. La información se recogió durante tres semanas, mediante entrevistas no estructuradas a fin de que tuviesen la libertad de expresar en sus propias palabras la situación que estaban viviendo en el colegio. El análisis de la información se realizó mediante la técnica concordancia interpretativa entre dos observadores en este caso con la colaboración de la psicóloga del Colegio y fueron organizados en un libro de Códigos mediante el programa ATLAS.TI

\section{Resultados}

La evaluación de los 52 estudiantes que constituían la población estudiantil total del Centro Educativo permitió identificar un grupo de 12 estudiantes que representa el 23\% del mismo, los que admitían haber recibido algún tipo de maltrato o intimidación, mientras otros 40, negaron haber sufrido maltrato alguno. Mediante entrevistas directas se procedio a depurar la muestra inicial, ya que siete de ellos mostraron resistencia a considerarse una víctima aduciendo que el maltrato era "un problema ya superado", era "una situación que no se había vuelto a repetir", o que "había ocurrido al inicio del año cuando no tenía amigos", por lo cual fueron descartados; sin embargo cinco de ellos (10\% de la población) reconocieron que dicha situación se presentaba frecuentemente y continuaban viviéndola en el momento presente (figura 1), los cuales pasaron a constituir la muestra definitiva para efectos del presente estudio. 


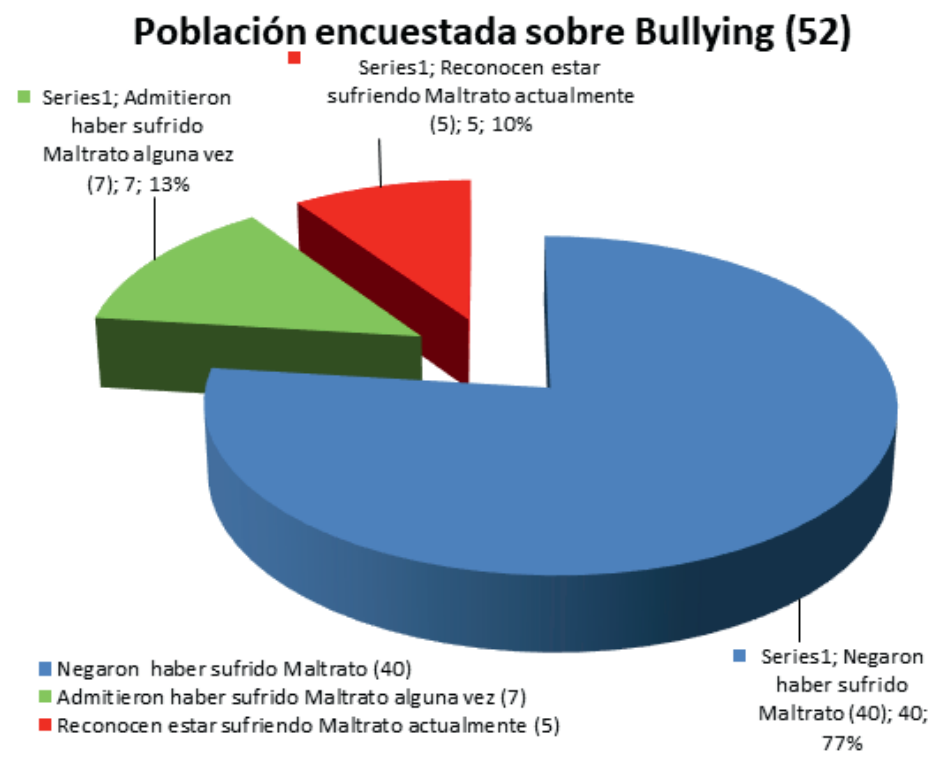

Figura 1. Porcentaje de estudiantes encuestados sobre Convivencia y Maltrato

Hay que aclarar que, mediante un muestreo intencionado se consideraron únicamente a aquellos estudiantes que respondieron con las alternativas (b) ó (c), en las preguntas del cuestionario. Luego se le convocó a una entrevista privada con la finalidad de indagar las circunstancias bajo las cuales ocurría el maltrato y la manera como ellos perciben dicha situación.

De estos cinco estudiantes, dos cursaban el 3er. año, ambos de sexo masculino y tres el 4 to. año, en este caso, dos de sexo masculino y uno femenino. Se encontró además que, en todos los casos, provenían de familias donde la autoridad era ejercida por la madre principalmente y el contacto con los padres era limitado por razones de trabajo.

Los datos fueron proporcionados de manera individual por cada uno de los estudiantes en entrevistas semiestructuradas frente a dos observadores que realizaban registros independientemente acerca de cómo estos estudiantes percibían sus relaciones interpersonales frente a la problemática del maltrato que reconocían estar viviendo y qué tipo de explicación o elaboración cognitiva daban a ello. Toda esta información registrada fue analizada, interpretada y posteriormente procesada con ayuda del programa ATLAS.TI para organizarla en un libro de códigos en base a cuatro categorías:

Resistencia y Enfado: Agrupaba expresiones con las que los estudiantes pretendían oponerse al acoso o señalaban un deseo de justicia externa.

Resignación: Expresiones que denotaban dimisión a un cambio o mejora en su situación, renunciando a cualquier forma de defensa.

Justificación: Manifestaciones verbales que pretendían ofrecer una explicación atenuante del maltrato sufrido, asumiendo culpabilidad en algunos casos.

Consuelo: Comentarios que a manera de reflexión asumían esperanza de un futuro justiciero que cambie la situación en que se encuentran. 
Tabla 1

Libro de Códigos de auto percepción de maltrato en alumnos víctimas de bullying

\begin{tabular}{|c|c|c|c|}
\hline Cod. & Definición & Expresiones & $\%$ \\
\hline RENFA & $\begin{array}{l}\text { Resistencia y } \\
\text { enfado }\end{array}$ & $\begin{array}{l}\text { "Ellos no van a lograr hacerme cambiar", "quisiera que se } \\
\text { mueran", "deberían expulsarlos del colegio" "Que se vayan al } \\
\text { infierno" ......"al diablo" .... "a la m". }\end{array}$ & 16.7 \\
\hline RESIG & Resignación & $\begin{array}{l}\text { "Siempre ha sido así", "no me importa", "pero nadie te ayuda", } \\
\text { "sería peor si me quejo", "es porque no sé defenderme", "en } \\
\text { todo sitio es así", "no me gusta hacer daño a nadie", "(qué } \\
\text { puedo hacer), son más bonitas que yo", "son mejores que yo", } \\
\text { "su papá es muy importante en el colegio, (no se puede hacer } \\
\text { nada)", "a nadie le importa" "ya se cansarán". }\end{array}$ & 38.9 \\
\hline JUSTO & Justificación & $\begin{array}{l}\text { "Yo los provoqué", "sólo lo hacen por bromear", "los estuve } \\
\text { mirando mucho y a nadie le gusta eso", "me causan gracia y } \\
\text { a veces me río de ellos", "lo hacen porque son los populares", } \\
\text { "los hice enojar mucho", "no debería ser tan bruto siempre los } \\
\text { hago rabiar", "si no fuese tan diferente no me molestarían" , } \\
\text { "yo debería cambiar", "es por q soy cobarde", "ya casi no me } \\
\text { molestan" "ellos tienen razón soy detestable". }\end{array}$ & 33.3 \\
\hline CONSU & Consuelo & $\begin{array}{l}\text { "Algún día me van a necesitar", "me van a envidiar algún día", } \\
\text { "yo voy a ser un profesional y se van a arrepentir", "algún día } \\
\text { voy a ser más fuerte". }\end{array}$ & 11.1 \\
\hline
\end{tabular}

Un análisis de las Categorías nos permitió identificar que el mayor porcentaje de incidencia de las expresiones acerca de la percepción de sus relaciones interpersonales estaba referido la "Resignación" (38.9\%) como elaboración cognitiva principal, seguido por la de "Resignación" (33.3\%); mientras que en menor porcentaje aparecen las de "Resistencia y enfado" (16.7\%) y la de "Consuelo" (11.1\%)
Asimismo, los estudiantes victimizados reportaron por lo menos 48 situaciones que pueden ser consideradas como acoso o maltrato, las cuales tuvieron como escenario cuatro lugares fundamentalmente: El Aula de clases, patio de recreo, pasillos del colegio y los baños. La evidencia en la figura 2.

Incidencia de Bullying por escenario

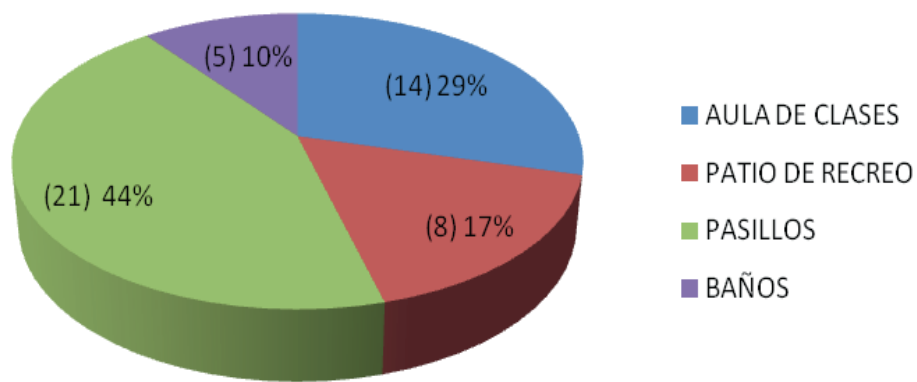

Figura 2. Cantidad de situaciones de Acoso o Maltrato percibidas de acuerdo al escenario de ocurrencia 
Finalmente, estas situaciones de Acoso o Maltrato manifestadas por los estudiantes victimas de Bullying, fueron agrupadas dentro de cuatro categorías semánticas basadas en las explicaciones que daban al problema; que incluían la marginación, hostigamiento, extorsión y agresión propiamente dicha.

Tabla 2

Libro de Códigos sobre las manifestaciones de Maltrato percibidas por un grupo de alumnos víctimas de bullying

\begin{tabular}{llll}
\hline Cod & Categoría & Manifestaciones & $\mathrm{N}^{\circ}$ \\
\hline MRG & Marginación & $\begin{array}{l}\text { Impedirle el ingreso, quitarle el habla, impedir su } \\
\text { participación en el aula o el recreo, discriminarlo }\end{array}$ & $\begin{array}{l}13 \\
(27 \%)\end{array}$ \\
HST & Hostigamiento & $\begin{array}{l}\text { Ridiculizarlo, esconderle sus pertenencias, menospreciarlo, } \\
\text { acusarlo en falso, burlarse, ponerle apodos, expresiones de } \\
\text { odio o desprecio. }\end{array}$ & $(46 \%)$ \\
EXT & Extorsión & $\begin{array}{l}\text { Amenazarlo con gestos o frases, chantajearlo con fotos o } \\
\text { videos, exigirle la entrega de pagos, prendas o alimentos }\end{array}$ & 5 \\
& Agresión & $\begin{array}{l}\text { Golpear o empujarlo, ponerle zancadillas, destruir o } \\
\text { malograr sus pertenencias, jalarle los cabellos, arrojarle } \\
\text { objetos, escupirle, gestos sexuales ofensivos. }\end{array}$ & $8(17 \%)$ \\
& &
\end{tabular}

Estas categorías organizadas de acuerdo a las características de las acciones que los estudiantes víctimas manifestaban y la manera cómo ellos las percibían, nos permitieron a su vez identificar el nivel de incidencia.

\section{Análisis y discusión}

Se ha podido observar que un $23 \%$ de la población estudiada manifiesta haber sufrido una situación de acoso o maltrato escolar en algún momento, sin embargo, únicamente el 10\% de la misma reconoce su condición de víctima actual, lo cual podría estar reflejando cierta resistencia a aceptar su realidad o una manera de paliar y salvaguardar su autoconcepto e integridad psicológica. Esto resulta particularmente entendible si consideramos que para nadie resulta atractivo tener problemas de interacción con sus compañeros de estudio o fracasar en sus relaciones con ellos y menos, verse expuesto bajo tal consideración.

Siendo el propósito del presente estudio conocer las razones que hacen que los estudiantes víctimas de Bullying en una escuela, permanezcan en esta situación sin intentar modificar su condición, perseveramos con ese 10\% para determinar la percepción que tienen sobre su problemática, siendo la mayor parte de sus expresiones catalogadas bajo la categoría de Resignación o Justificación de su condición de víctima. Esto pone de manifiesto importantes consideraciones a tomar en cuenta en los programas de intervención que pudieran estructurarse, puesto que esta actitud que las víctimas de bullying suelen asumir, podrían reforzar y hasta promover el comportamiento de los agresores en la medida que fomenta la impunidad.

No debemos desestimar, como datos adicionales que arroja el presente estudio, el tipo de Acoso o Maltrato escolar más proclives y las características de los escenarios que aparentemente más propicios para el desarrollo del Bullying, cuyo conocimiento favorecería la aplicación de programas preventivos realmente eficaces. 


\section{Conclusiones}

El presente estudio nos ha permitido obtener una visión mucho más realista del fenómeno del Bullying, como característica de la problemática educativa contemporánea, al proporcionarnos valiosa información desde la perspectiva de las propias víctimas de este maltrato. Los estudiantes víctimas de bullying, difícilmente suelen reconocer frente a otros esta condición buscando por lo general alguna justificación que permita atenuar sus efectos sobre su ya maltratada autoestima. Los primeros argumentos a los que recurren para contrarrestar ello, suelen ser la negación de su ocurrencia, buscando explicaciones incidentales que le permitan mantener una imagen de competencia social, eludiendo la evidencia de los hechos y delegando responsabilidades en otras personas o situaciones. El hecho es que, el aceptarse como víctima implica además del maltrato, ser tipificado como diferente y por ende aceptar el vacío social en su entorno, lo cual atenta con la necesidad de interacción constante de todo ser humano que le permite, a lo largo de su vida, establecer un proceso de socialización fundamental para su desarrollo emocional.

Por otro lado, cuando reconocen su condición como víctimas de bullying, muestran una serie de elaboraciones cognitivas que tienen en función de la interpretación su realidad, las cuales van desde los deseos o esperanzas de justicia futura hasta la resignación y renuncia a toda defensa. Es justamente esta última posición la que resulta ser la más típica o constante, lo cual es en extremo peligrosa, dado que lleva al estudiante víctima del maltrato a justificar a sus agresores e inclusive culparse a sí mismo por tal situación, lo que reflejaría ya, serias perturbaciones de su personalidad.

Por tanto, es necesario contemplar esta situación en los programas de intervención del bullying, especialmente en relación al fortalecimiento del autoestima y desarrollo de resiliencia en las víctimas del maltrato. Por último, se ratifica conforme a la lógica, que los escenarios más propicios para el acoso escolar resultan aquellos donde por lo general no hay supervisión adulta, como los pasillos y alrededores, aunque existen otros lugares como las aulas y el mismo patio de recreo que no dejan de tener importancia. El caso de los baños probablemente debido a que los colegios incluyen personal de limpieza perenne ha bajado en importancia. Es menester mencionar que, si bien las cifras o porcentajes representativos en cuanto al tipo de Acoso o Maltrato señalan en primer lugar al Hostigamiento y extorsión y en segundo lugar a las agresiones, ello puede no tener suficiente propiedad de generalización por ser un análisis cuantitativo frente a una muestra reducida.

Es claro que el bullying afecta seriamente el proceso de socialización de los estudiantes y más seriamente el desarrollo de su personalidad, por ello y acorde con la perspectiva mostrada en el presente estudio, consideramos importante señalar que las acciones de intervención frente al Bullying sean llevadas a cabo mediante programas de integrales, que involucren tanto a las víctimas como a los acosadores y los diferentes participantes en la problemática del Acoso y Maltrato Escolar, llámese espectadores, padres de familia, profesores etc. 


\section{Referencias}

Avilés, J.M. (2001). La Intimidación y maltrato en los centros escolares (Bullying). Bilbao: Osasuna.

Benites, L., Carozzo, J., Horna, V., Palomino, L., Salgado, C., Uribe, C. \& Zapata, L. (2012). Bullying y Convivencia en la Escuela. Aspectos conceptuales, aplicativos y de investigación. Lima: Dennis Morzán.

Carozzo, J. (2010). El bullyng en la escuela. Rev. Psicol. 12, 329-346. Recuperado de http:// sisbib.unmsm.edu.pe/BVRevistas/rev_ psicologia_cv/v12_2010/pdf/a13.pdf.

Carozzo, J., Benites, L., Zapata, L. \& Horna, V. (2012). El Bullying no es juego. Guía para todos. Lima: Dennis Morzán.

Cerezo, R. (2001). Variables de personalidad asociadas en la dinámica bullying (agresores versus víctimas) en niños y niñas de 10 a 15 años. Anales de Psicología, 17(1), 37-43.

Del Barrio, M.E., Montero, I., Gutiérrez, H. y Fernández, I. (2003). La realidad del maltrato entre iguales en los centros de secundaria españoles. Infancia y Aprendizaje, 26(1), 25-47.

Díaz, M. (2000). Convivencia escolar y prevención de la Violencia. Madrid: Universidad Complutense.
Fernández, I. (1996). Manifestaciones de la violencia en la escuela. El clima escolar. Revista de renovación pedagógica. Educadores, 180, 35-54.

Giraldo, L. \& Mera, R. (2000). Clima escolar: Percepción del estudiante. Medellín. 31, 23-27.

Ley General de Educación, Nro. 28044. Art. 66).

Monjas, M ${ }^{a}$ I. (1992). La competencia social en la edad escolar. Diseño, aplicación y validación del Programa de Habilidades de Interacción Social (Tesis Doctoral). Universidad de Salamanca. España.

Oliveros, M., Figueroa, L., Mayorga, G., Cano, G., Quispe, Y., Barrientos, A. (2009). Factores de riesgo de violencia escolar (bullying) severa en colegios privados de tres zonas de la sierra del Perú. Anales Facultad de medicina, 70(4), 255-8. Lima: UNMSM

Olweus, D. (1998). Conductas de acoso y amenaza entre escolares. Madrid: Morata.

Ortega, R. \& Mora-Merchán, J. (1996). El aula como escenario de la vida afectiva y moral. Cultura y Educación, 3, 5-18. Recuperado de https://doi.org/10.1174/113564096763270280

Smith, P. K. y Thompson, D. (1991). Practical Aproaches lo Bullying. London: David Fulton Publishers. 


\section{Apéndice}

\section{Ficha sociodemográfica}

Nombre

Edad:

Sexo:

Centro Educativo:

Nombre del Padre:

Edad:

Nombre de la madre:

Edad:

Hermanos:

Nombre:

Edad:

Nombre:

Edad:

Nombre:

Edad:

Lugar que ocupa entre los hermanos:

¿Con quién vives?

¿Cómo te llevas con tus padres?

¿Cómo te llevas con tus hermanos?

¿Quién castiga en casa?

¿De qué manera te castigan cuando te portas mal?: 


\section{Cuestionario sobre convivencia y maltrato}

Edad.

Grado estudios

Sexo.

A continuación te presentamos una serie de situaciones que te pueden haber ocurrido. MARCA las respuestas que consideres describen el trato que recibes en el colegio.

1.- ¿Cómo te llevas con la mayoría de tus compañeros?

a.- Bien

b.- Ni bien, ni mal

C.- Mal

2.- ¿Qué cantidad de amigos tienes?

a.- Muchos

b.- Pocos

c.- Ninguno

3.- ¿Alguna vez tus compañeros no han querido estar contigo?

a.- Nunca

b.- Algunas veces

c.- Muchas veces

4.- ¿Este año, te ha intimidado o maltratado alguno de tus compañeros (as)?

a.- Nunca

b.- Algunas veces

c.- Muchas veces o casi todos los días

5.- ¿Cómo te sientes en el Colegio?

a.- Bien

b.- Ni bien, ni mal

c.- Mal 